\title{
Sensitivity Analysis of the Dimensionless Parameters in Scaling a Polymer Flooding Reservoir
}

\author{
Yuhu Bai · Jiachun Li · Jifu Zhou • Qingping Li
}

Received: 29 August 2006 / Accepted: 12 July 2007 / Published online: 12 October 2007

(C) Springer Science+Business Media B.V. 2007

\begin{abstract}
A set of scaling criteria of a polymer flooding reservoir is derived from the governing equations, which involve gravity and capillary force, compressibility of water, oil, and rock, non-Newtonian behavior of the polymer solution, absorption, dispersion, and diffusion, etc. A numerical approach to quantify the dominance degree of each dimensionless parameter is proposed. With this approach, the sensitivity factor of each dimensionless parameter is evaluated. The results show that in polymer flooding, the order of the sensitivity factor ranges from $10^{-5}$ to $10^{0}$ and the dominant dimensionless parameters are generally the ratio of the oil permeability under the condition of the irreducible water saturation to water permeability under the condition of residual oil saturation, density, and viscosity ratios between water and oil, the reduced initial oleic phase saturation and the shear rate exponent of the polymer solution. It is also revealed that the dominant dimensionless parameters may be different from case to case. The effect of some physical variables, such as oil viscosity, injection rate, and permeability, on the dominance degree of the dimensionless parameters is analyzed and the dominant ones are determined for different cases.
\end{abstract}

Keywords Polymer flooding $\cdot$ Dimensionless parameter $\cdot$ Sensitivity analysis $\cdot$ Multiphase percolation

\section{Introduction}

Most of the oil fields in China have been developed by water flooding, but the recovery efficiency is presently very low and the water cut is over $80 \%$ (Han et al. 1999). Only 30-40\% of the original oil reserves can be recovered after completing the primary and secondary

Y. Bai $(\bowtie) \cdot$ Q. Li

Technology Research Department, CNOOC Research Center, Beijing, 100027, China e-mail: byh_2002@163.com

J. Li · J. Zhou

Division of Engineering Sciences, Institute of Mechanics, Chinese Academy of Sciences, Beijing, 100080, China 
recoveries (Shen 2003). The residual oil remain in the strata as an oil film on the surface of the pores and stagnant oil in the blind ends, throats and some places is not swept by water flooding. The forces holding the remaining oil include interfacial tension among oil, water, and gas phases and viscous force, etc. (Wang et al. 2000). To enhance oil recovery, polymer flooding as a major enhancement method is widely carried out in China. On the one hand, the addition of polymer to the water flooding increases the viscosity of the aqueous phase to adapt the oil viscous force. On the other hand, the polymer solution reduces the permeability of the aqueous phase. These two factors result in an improvement in the mobility ratio between aqueous and oleic phases, increasing the areal and volumetric sweep efficiency. The field and laboratory experiments have proved that polymer flooding can enhance oil recovery by 10-20\% (Lu etal. 1996).

Physical simulation plays an important role in revealing the mechanism of the driving process and optimizing the development programs in the short term at a low cost. The principle of similarity or scaling law is crucial for physical simulations (Sedov 1959). A set of scaling criteria for polymer, emulsion, and foam flooding experiments was obtained by inspectional analysis (Islam and Farouq Ali 1989). They focused on the complicated flooding processes involving mass transfer among phases, the interfacial tension, diffusion, dispersion, absorption, and mechanical entrapment. The scaling criteria of surfactant-enhanced alkaline/polymer multiple flooding flows was gained, which involved transient interfacial tension and non-equilibrium mass transfer phenomena (Islam and Farouq Ali 1990). They observed that in most cases the mass transfer rate in the model is different from that in the prototype. However, both rates were assumed to be the same as in previous studies. Though some experiments (Wang and Han 1994; Sorbie etal. 1985) have been conducted to reveal the driving mechanisms in the polymer flooding process, few physical simulations are based on the principle of similarity. The reason is that the physical simulation of polymer flooding associates with such complicated factors as convection, dispersion, diffusion, entrapment, and non-Newtonian behavior, which leads to so many dimensionless parameters that cannot be simultaneously satisfied. The precise scaling of transverse dispersion coupled with the requirement of geometric similarity would impractically require a large model and a very long time interval for miscible experiments (Pozzi and Blackwell 1963). In some cases, the geometry similarity and the gravity force have to be relaxed in order to satisfy the requirement of the miscible displacement. Simultaneously, modeling the ratios of the capillary and the driving forces to the gravity force would induce a considerably large permeability for a water flooding experiment, which is very difficult to realize in the laboratory (Shen 2000).

Though numerous literature has focused on the displacement mechanism (Prabir and Pasa 2004; Kevin et al. 1998), numerical simulation (Luis et al. 2005), field projection design and prediction technique (Wang et al. 1993; Delamaide et al. 1994), few papers have reported on the similarity criteria. Generally speaking, there are so many parameters involved in the process of polymer flooding flows. Therefore, it is very difficult or sometimes even impossible to keep all the similarity parameters identical in the laboratory experiment. To tackle this kind of problem, an efficient and practical way out is to single out the dominant parameters and to relax the secondary ones in laboratory experiments. Theoretically arranging all the dimensionless parameters in the order of importance is not easy or even impossible to realize. In our previous work, a numerical approach of sensitivity analysis of dimensionless parameters is proposed based on the scaling criteria of water flooding flow (Bai et al. 2005). With this approach, the dominance degree of the dimensionless parameters can be quantitatively evaluated and the dominant ones can be conveniently singled out.

In the following sections, we first derive the scaling criteria of polymer flooding involving the gravity force, the capillary force, the compressibility of water, oil, and rock, the 
non-Newtonian behavior of the polymer solution, absorption, convection, dispersion, and diffusion. Then, with the numerical approach of sensitivity analysis, we quantify the dominance degree of all the dimensionless parameters and single out the dominant ones. Finally, we analyze the effect of physical variables on the dominance degree of the dimensionless parameters.

\section{The Governing Equations}

The process of polymer flooding is actually the flow of two phases, the oleic and the aqueous phases. The oleic phase contains only oil and the aqueous phase contains water and polymer. Therefore, the mass transfer between water and oil or polymer and oil is neglected. In addition, the following assumptions are made: (1) Isothermal flow occurs in a homogeneous and isotropic medium; (2) Darcy's and Fick's laws are valid; (3) The polymer only reduces the permeability of the aqueous phase and has no effect on the permeability of the oleic phase; (4) The aqueous and oleic phases and the rock are slightly compressible. Therefore, the governing equations based on a quarter of a five-spot well pattern are as follows.

The mass balance equation of the oleic phase is

$$
\frac{\partial\left(\phi s_{o} \rho_{o}\right)}{\partial t}=\nabla \cdot\left(\frac{\rho_{o}}{\mu_{o}} k_{o} \nabla\left(p_{o}+\rho_{o} g \Delta z\right)\right)+q_{o} .
$$

The mass balance equation of the aqueous phase is given by

$$
\frac{\partial\left(\phi s_{a} \rho_{a}\right)}{\partial t}=\nabla \cdot\left(\frac{\rho_{a}}{\mu_{a}} k_{a} \nabla\left(p_{a}+\rho_{a} g \Delta z\right)\right)+q_{a} .
$$

The equation of polymer transport is written as

$$
\begin{aligned}
& \frac{\partial\left(\phi s_{a} \rho_{a} C_{a p}\right)}{\partial t}+\frac{\partial\left(\rho_{r}(1-\phi) \rho_{a} \hat{C}_{a p}\right)}{\partial t} \\
& =\nabla \cdot\left(D \phi s_{a} \nabla\left(C_{a p} \rho_{a}\right)\right)+\nabla \cdot\left(C_{a p} \frac{\rho_{a} k_{a}}{\mu_{a}} \nabla\left(p_{a}+\rho_{a} g \Delta z\right)\right)+q_{a} C_{a w p} .
\end{aligned}
$$

Here

$$
\begin{aligned}
& q_{o}=\frac{\pi \rho_{o} k_{o}\left(p_{w f}-p_{o}\right)}{2 \mu_{o} \ln r_{e o} / r_{o}} \delta\left(x-x_{p}\right) \delta\left(y-y_{p}\right), \\
& q_{a}=\frac{\rho_{a} q_{I}}{4 h} \delta(x) \delta(y)+\frac{\pi \rho_{a} k_{a}\left(p_{w f}-p_{a}\right)}{2 \mu_{a} \ln r_{e o} / r_{o}} \delta\left(x-x_{p}\right) \delta\left(y-y_{p}\right) .
\end{aligned}
$$

$p, \mu, \rho, K$, and $s$ mean the pressure, the density, the viscosity, the effective permeability, and saturation, subscripts $w$ and $o$ indicate aqueous and oleic phases, respectively. $p_{w f}$ is the bottom pressure of the production well, $\phi$ the porosity of rock, $g$ the gravitational acceleration, $x_{p}$ and $y_{p}$ the coordinates of the production well, $q_{I}$ the injection rate, $r_{\mathrm{o}}$ the well radius, $C_{a p}$ the polymer concentration in aqueous phase, $\hat{C}_{a p}$ the amount of polymer adsorbed per unit mass of rock, $C_{a w p}$ the polymer concentration in the production well, $D$ the apparent dispersion coefficient, and $\rho_{r}$ the density of rock.

The saturation relation reads:

$$
s_{o}+s_{a}=1 .
$$


The capillary force equation can be expressed as

$$
p_{c}=p_{o}-p_{a}=\sigma \cos \theta \sqrt{\phi / K} J\left(s_{a}\right),
$$

in which $p_{c}$ is the capillary force, $K$ the absolute permeability, $\sigma, \theta$ the interfacial tension and the contact angle between the aqueous and oleic phases, and $J\left(s_{a}\right)$ the capillary force function.

The state equations are

$$
\begin{aligned}
\rho_{o} & =\rho_{o 0}\left(1+C_{o}\left(p_{o}+\rho_{o 0} g z-p_{o 0}\right)\right), \quad \rho_{a}=\rho_{a 0}\left(1+C_{a}\left(p_{a}+\rho_{a 0} g z-p_{a 0}\right)\right), \\
\phi & =\phi_{0}\left(1+C_{\phi}\left(\frac{p_{a}+p_{o}+\rho_{o 0} g z+\rho_{a 0} g z}{2}-\frac{p_{a 0}+p_{o 0}}{2}\right)\right),
\end{aligned}
$$

in which the symbols have the same meaning as above. Subscript 0 indicates physical quantities at a certain condition, $C_{o}, C_{a}$, and $C_{\phi}$ the compressibility of oil, water, and rock, respectively.

Initial conditions are given as

$$
p_{o}(x, y, z, 0)=p_{o i}, \quad s_{o}(x, y, z, 0)=s_{o i}, \quad C_{a p}(x, y, z, 0)=C_{a p i},
$$

and boundary conditions are

$$
\rho_{o} v_{o n}=-\frac{\rho_{o}}{\mu_{o}} k_{o} \nabla\left(p_{o}+\rho_{o} g \Delta z\right)=0, \quad \rho_{a} v_{a n}=-\frac{\rho_{a}}{\mu_{a}} k_{a} \nabla\left(p_{a}+\rho_{a} g \Delta z\right)=0 .
$$

It should be noted that with the polymer mixed, the properties of the aqueous phase, such as permeability, viscosity, dispersion, adsorption, and constitutive relation, etc., might change. Therefore, some factors should be particularly considered with some additional equations supplied.

\subsection{Permeability Reduction Factor}

Polymer reduces the permeability of the aqueous phase, but does not change the permeability of the oleic phase. This increases the flow resistance of the polymer solution in porous media, improving the sweep efficiency and thus enhancing oil recovery. The permeability reduction factor defined as $R_{k}=k_{w} / k_{a}$ denotes the effect of polymer on the permeability of aqueous phase. It is always less than unit, indicating the permeability reduction of the aqueous phase. It is proportional to $\hat{C}_{a d}$, the adsorption amount of polymer, and can be expressed as (Islam and Farouq Ali 1989)

$$
R_{k}=1+\left(R_{k \max }-1\right) \frac{\hat{C}_{a d}}{\hat{C}_{a d \max }},
$$

in which $R_{k \max }$ is the maximum permeability reduction factor and $\hat{C}_{a d \max }$ the maximum adsorption amount per unit mass of rock.

\subsection{The Adsorption of Polymer in Porous Media}

The adsorption of polymer due to mechanical entrapment, chemical adsorption, and retention causes a reduction in the permeability of the aqueous phase. This behavior can be described with the Langmuir adsorption isotherm (Littmann 1991) as follows:

$$
\frac{\hat{C}_{a d}}{\hat{C}_{a d \max }}=\frac{b C_{a p}}{1+b C_{a p}},
$$


in which $b$ is a constant. Therefore, the adsorption term on the left-hand side in the polymer governing equation can be rewritten as

$$
\frac{\partial\left(\rho_{r}(1-\phi) \rho_{a} \hat{C}_{a p}\right)}{\partial t}=\frac{\rho_{r} b \hat{C}_{a d \max }}{\left(1+b C_{a p}\right)^{2}} \frac{\partial\left((1-\phi) \rho_{a} C_{a p}\right)}{\partial t} .
$$

\subsection{Viscosity Expression of the Polymer Solution}

The viscosity of the polymer solution can be expressed as (Wang 1990)

$$
\mu_{a}=\mu_{w}\left(1+\left(\frac{\gamma}{\gamma_{\min }}\right)^{n-1}\left(\frac{C_{s}}{C_{s \min }}\right)^{m}\left(A_{p 1} C_{a p}+A_{p 2} C_{a p}^{2}+A_{p 3} C_{a p}^{3}\right)\right),
$$

where $\mu_{w}$ is the viscosity of water, $\gamma$ the shear rate of the polymer solution, $\gamma_{\min }$ the minimum shear rate within the range of pseudoplastic fluid, $n$ the shear rate exponent, $m$ the salinity exponent, $C_{s}$ the salinity, $C_{s}$ min the minimum salinity affecting the viscosity of polymer solution, and $A_{p 1}, A_{p 2}$, and $A_{p 3}$ the constants. If the salinity is not considered, $m=0$. The viscosity equation reads

$$
\mu_{a}=\mu_{w}\left(1+\left(\frac{\gamma}{\gamma_{\min }}\right)^{n-1}\left(A_{p 1} C_{a p}+A_{p 2} C_{a p}^{2}+A_{p 3} C_{a p}^{3}\right)\right) .
$$

\subsection{Shear Rate Expression}

The polymer solution is a kind of non-Newtonian fluid. Its viscosity is related to the shear rate. The shear rate considering the permeability reduction effect is given as (Wang et al. 1992)

$$
\gamma=\left(\frac{3 n+1}{4 n}\right)^{\frac{n}{n-1}} \frac{v_{a}}{\sqrt{0.5 c^{\prime} k k_{r w} \phi s_{w} / R_{k}}}
$$

where $v_{a}$ denotes the percolation velocity of the aqueous phase, and $c^{\prime}$ is the tortuosity ranging from $25 / 12$ to 2.5 .

\subsection{Dispersion Coefficient Expression}

The dispersion consists of molecular diffusion and mechanical dispersion due to flows (Perkins and Johnston 1962). Its expression is described as follows

$$
D=\frac{D_{0}}{F \phi}+0.5 v_{a} \delta d_{p}, \quad\left(\frac{v_{a} \delta d_{p}}{D_{0}}<50\right),
$$

where $D_{0}$ denotes the molecular diffusion coefficient, $F$ the formation electrical resistivity factor, $d_{p}$ the mean particle diameter in porous media, and $\delta$ the inhomogeneity of the formation, always larger than unit and about $\delta=3.5$ for the typical random distribution.

\section{The Scaling Criteria}

We introduce the dimensionless independent variables

$$
x_{D}=\frac{x}{x_{R}}, \quad y_{D}=\frac{y}{y_{R}}, \quad z_{D}=\frac{z}{z_{R}}, \quad t_{D}=\frac{q_{I} t}{\phi x_{R} y_{R} h\left(1-s_{c w}-s_{r o}\right)}=\frac{q_{I} t}{\phi x_{R} y_{R} h \Delta s},
$$


the dimensionless dependent variables

$$
p_{a D}=\frac{p_{a} K_{\text {row }} h}{q_{I} \mu_{w}}, \quad p_{o D}=\frac{p_{o} K_{\text {row }} h}{q_{I} \mu_{w}}, \quad C_{a p D}=\frac{C_{a p}}{C_{a w p}},
$$

some other dimensionless parameters

$$
\begin{aligned}
& \mu_{o D}=\frac{\mu_{o}}{\mu_{w}}, \quad \rho_{o 0 D}=\frac{\rho_{o 0}}{\rho_{a 0}}, \quad C_{o D}=\frac{C_{o} q_{I} \mu_{w}}{K_{\text {row }} h}, \quad C_{a D}=\frac{C_{a} q_{I} \mu_{w}}{K_{\text {row }} h}, \\
& C_{\phi D}=\frac{C_{\phi} q_{I} \mu_{w}}{K_{\text {row }} h}, \quad p_{a 0 D}=\frac{p_{a 0} K_{\text {row }} h}{q_{I} \mu_{w}}, \quad p_{o 0 D}=\frac{p_{o 0} K_{\text {row }} h}{q_{I} \mu_{w}}, \quad p_{o i D}=\frac{p_{o i} K_{\text {row }} h}{q_{I} \mu_{w}}, \\
& p_{w f D}=\frac{p_{w f} K_{\text {row }} h}{q_{I} \mu_{w}}, \quad r_{e o D}=\frac{r_{e o}}{x_{R}}, \quad r_{o D}=\frac{r_{o}}{x_{R}}, \quad C_{\text {iapD }}=\frac{C_{\text {iap }}}{C_{a w p}}, \quad \bar{s}_{\text {oi }}=\frac{s_{o i}-s_{\text {ro }}}{\Delta s}
\end{aligned}
$$

and normalized saturation and permeability

$$
\bar{s}_{a}=\frac{s_{a}-s_{c w}}{\Delta s}, \quad \bar{s}_{o}=\frac{s_{o}-s_{r o}}{\Delta s}, \quad K_{o D}=\frac{K_{o}}{K_{c w o}}, \quad K_{w D}=\frac{K_{w}}{K_{\text {row }}} .
$$

In the above expressions, $x_{R}, y_{R}$, and $z_{R}$ are reference lengths in three directions, respectively. $s_{r o}$ and $s_{c w}$ denote the residual oil saturation and the irreducible water saturation, respectively. $K_{c w o}$ refers to the effective permeability of the oil phase under the condition of irreducible water saturation. $K_{\text {row }}$ the effective permeability of the water phase under the condition of residual oil saturation, and the subscript $D$ means a dimensionless parameter. Substituting all these variables into the governing equations, state equations, supplementary equations and the initial and boundary conditions, yields the following equations and expressions.

The dimensionless equation of mass balance of the oleic phase is derived as

$$
\begin{aligned}
\pi_{4} \pi_{1} & \frac{\partial}{\partial x_{D}}\left(\rho_{o D} \frac{K_{o D}}{\mu_{o D}} \frac{\partial p_{o D}}{\partial x_{D}}\right)+\frac{\pi_{1}}{\pi_{4}} \frac{\partial}{\partial y_{D}}\left(\rho_{o D} \frac{K_{o D}}{\mu_{o D}} \frac{\partial p_{o D}}{\partial y_{D}}\right) \\
& +\pi_{4} \pi_{5}^{2} \pi_{1} \frac{\partial}{\partial z_{D}}\left(\rho_{o D} \frac{K_{o D}}{\mu_{o D}} \frac{\partial p_{o D}}{\partial z_{D}}\right)+\pi_{16} \pi_{1} \pi_{4} \pi_{5}^{2} \frac{\partial}{\partial z_{D}}\left(\rho_{o D}^{2} \rho_{o 0 D} \frac{K_{o D}}{\mu_{o D}}\right) \\
& +\pi_{1} \rho_{o D} \frac{\pi k_{o D}\left(p_{w f D}-p_{o D}\right)}{\mu_{o D} 2 \ln r_{o O} / r_{o D}} \delta\left(x_{D}-\pi_{6}\right) \delta\left(y_{D}-\pi_{7}\right) \\
= & \frac{\partial\left(\rho_{o D} \phi_{D} \bar{s}_{o}\right)}{\partial t_{D}}+\pi_{11} \frac{\partial\left(\rho_{o D} \phi_{D}\right)}{\partial t_{D}} .
\end{aligned}
$$

The dimensionless equation of mass balance of aqueous phase is given as

$$
\begin{aligned}
\frac{\pi_{4}}{\pi_{25}} & \frac{\partial}{\partial x_{D}}\left(\frac{\rho_{a D} K_{w D}}{R_{k D} \mu_{a D}} \frac{\partial p_{a D}}{\partial x_{D}}\right)+\frac{1}{\pi_{4} \pi_{25}} \frac{\partial}{\partial y_{D}}\left(\frac{\rho_{a D} K_{w D}}{R_{k D} \mu_{a D}} \frac{\partial p_{a D}}{\partial y_{D}}\right) \\
& +\frac{\pi_{4} \pi_{5}^{2}}{\pi_{25}} \frac{\partial}{\partial z_{D}}\left(\frac{\rho_{a D} K_{w D}}{R_{k D} \mu_{a D}} \frac{\partial p_{a D}}{\partial z_{D}}\right)+\frac{\pi_{16} \pi_{4} \pi_{5}^{2}}{\pi_{25}} \frac{\partial}{\partial z_{D}}\left(\rho_{a D}^{2} \frac{K_{w D}}{R_{k D} \mu_{a D}}\right) \\
& +\rho_{a D}\left[\frac{1}{4} \delta\left(x_{D}\right) \delta\left(y_{D}\right)+\frac{1}{\pi_{25}} \frac{\pi k_{w D}\left(p_{w f D}-p_{a D}\right)}{R_{k D} \mu_{a D} 2 \ln r_{e o D} / r_{o D}} \delta\left(x_{D}-\pi_{6}\right) \delta\left(y_{D}-\pi_{7}\right)\right] \\
= & \frac{\partial\left(\rho_{a D} \phi_{D} \bar{s}_{a}\right)}{\partial t_{D}}+\pi_{10} \frac{\partial\left(\rho_{a D} \phi_{D}\right)}{\partial t_{D}} .
\end{aligned}
$$


The dimensionless transport equation of polymer component reads

$$
\begin{aligned}
& \frac{\partial}{\partial x_{D}}\left(\left(\bar{s}_{a} D_{x D 1}+D_{x D 2}\right) \frac{\partial}{\partial x_{D}}\left(C_{a p D} \rho_{a D}\right)\right)+\frac{\partial}{\partial y_{D}}\left(\left(\bar{s}_{a} D_{y D 1}+D_{y D 2}\right) \frac{\partial}{\partial y_{D}}\left(C_{a p D} \rho_{a D}\right)\right) \\
& +\frac{\partial}{\partial z_{D}}\left(\left(\bar{s}_{a} D_{z D 1}+D_{z D 2}\right) \frac{\partial}{\partial z_{D}}\left(C_{a p D} \rho_{a D}\right)\right)+\frac{\pi_{4}}{\pi_{25}} \frac{\partial}{\partial x_{D}}\left(\frac{\rho_{a D} C_{a p D} K_{w D}}{R_{k D} \mu_{a D}} \frac{\partial p_{a D}}{\partial x_{D}}\right) \\
& \quad+\frac{1}{\pi_{4} \pi_{25}} \frac{\partial}{\partial y_{D}}\left(\frac{\rho_{a D} C_{a p D} K_{w D}}{R_{k D} \mu_{a D}} \frac{\partial p_{a D}}{\partial y_{D}}\right) \\
& +\frac{\pi_{4} \pi_{5}^{2}}{\pi_{25}} \frac{\partial}{\partial z_{D}}\left(\frac{\rho_{a D} C_{a p D} K_{w D}}{R_{k D} \mu_{a D}} \frac{\partial p_{a D}}{\partial z_{D}}+\pi_{16} \rho_{a D}^{2} \frac{C_{a p D} K_{w D}}{R_{k D} \mu_{a D}}\right) \\
& +\rho_{a D}\left[\frac{1}{4} \delta\left(x_{D}\right) \delta\left(y_{D}\right)+\frac{1}{\pi_{25}} \frac{\pi C_{a p D} k_{w D}\left(p_{w f D}-p_{a D}\right)}{2 R_{k D} \mu_{a D} \ln r_{e o D} / r_{o D}} \delta\left(x_{D}-\pi_{6}\right) \delta\left(y_{D}-\pi_{7}\right)\right] \\
& =\frac{\partial\left(\rho_{a D} \phi_{D} \bar{s}_{a} C_{a p D}\right)}{\partial t_{D}}+\pi_{10} \frac{\partial\left(\rho_{a D} \phi_{D} C_{a p D}\right)}{\partial t_{D}} \\
& +\frac{\pi_{26}}{\left(1+\pi_{28} C_{a p D}\right)^{2}} \frac{\partial\left(C_{a p D} \rho_{a D}\right)}{\partial t_{D}}-\frac{\pi_{27}}{\left(1+\pi_{28} C_{a p D}\right)^{2}} \frac{\partial\left(C_{a p D} \phi_{D} \rho_{a D}\right)}{\partial t_{D}},
\end{aligned}
$$

where

$$
\begin{aligned}
& \rho_{a D}=1+C_{a D}\left(p_{a D}-p_{a 0 D}\right)+\pi_{16} C_{a D} z_{D}, \\
& \rho_{o D}=1+C_{o D}\left(p_{o D}-p_{o 0 D}\right)+\pi_{16} C_{o D} \rho_{o 0 D} z_{D}, \\
& \phi_{D}=1+C_{\phi D}\left(\frac{p_{a D}+p_{o D}}{2}-\frac{p_{a 0 D}+p_{o 0 D}}{2}\right)+\frac{\pi_{16}}{2} \rho_{o 0 D} C_{\phi D} z_{D}+\frac{\pi_{16}}{2} C_{\phi D} z_{D}, \\
& D_{x D 1}=\pi_{29} \pi_{4}-\pi_{30} \pi_{4} \frac{0.5 k_{w D} \phi_{D}}{R_{k D} \mu_{a D}} \frac{\partial p_{a D}}{\partial x_{D}}, \\
& D_{x D 2}=\pi_{29} \pi_{10} \pi_{4}-\pi_{30} \pi_{10} \pi_{4} \frac{0.5 k_{w D} \phi_{D}}{R_{k D} \mu_{a D}} \frac{\partial p_{a D}}{\partial x_{D}}, \\
& D_{y D 1}=\frac{\pi_{29}}{\pi_{4}}-\frac{\pi_{30}}{\pi_{4}^{2}} \frac{0.5 k_{w D} \phi_{D}}{R_{k D} \mu_{a D}} \frac{\partial p_{a D}}{\partial y_{D}}, \\
& D_{y D 2}=\frac{\pi_{29} \pi_{10}}{\pi_{4}}-\frac{\pi_{30} \pi_{10}}{\pi_{4}^{2}} \frac{0.5 k_{w D} \phi_{D}}{R_{k D} \mu_{a D}} \frac{\partial p_{a D}}{\partial y_{D}}, \\
& D_{z D 1}=\pi_{29} \pi_{4} \pi_{5}^{2}-\pi_{30} \pi_{4} \pi_{5}^{3} \frac{0.5 k_{w D} \phi_{D}}{R_{k D} \mu_{a D}} \frac{\partial p_{a D}}{\partial z_{D}}-\pi_{16} \pi_{30} \pi_{4} \pi_{5}^{3} \frac{0.5 k_{w D} \rho_{a D} \phi_{D}}{R_{k D} \mu_{a D}}, \\
& D_{z D 2}=\pi_{29} \pi_{4} \pi_{5}^{2} \pi_{10}-\pi_{30} \pi_{4} \pi_{5}^{3} \pi_{10} \frac{0.5 k_{w D} \phi_{D}}{R_{k D} \mu_{a D}} \frac{\partial p_{a D}}{\partial z_{D}}-\pi_{16} \pi_{30} \pi_{4} \pi_{5}^{3} \pi_{10} \frac{0.5 k_{w D} \rho_{a D} \phi_{D}}{R_{k D} \mu_{a D}} .
\end{aligned}
$$

The dimensionless saturation relation is

$$
\bar{s}_{o}+\bar{s}_{a}=1 .
$$

The dimensionless capillary force can be expressed as

$$
p_{c D}=\left(p_{o D}-p_{w D}\right)=\pi_{13} \sqrt{\phi_{D}} \pi_{24} .
$$

The dimensionless initial conditions are

$$
p_{o D}=p_{o i D}\left(x_{D}, y_{D}, z_{D}, 0\right), \quad \bar{s}_{o}=\bar{s}_{o i}\left(x_{D}, y_{D}, z_{D}, 0\right), \quad C_{a p D}=C_{a p i D}\left(x_{D}, y_{D}, z_{D}, 0\right),
$$


and the dimensionless boundary conditions are specified as

$$
\frac{\partial p_{l D}}{\partial x_{D}}=0, \quad \frac{\partial p_{l D}}{\partial y_{D}}=0, \quad \frac{\partial p_{l D}}{\partial z_{D}}+\pi_{16} \rho_{a D}=0 .
$$

The dimensionless viscosity equation of the polymer solution is given as follows

$$
\mu_{a D}=\left(1+\left(\frac{\gamma}{\gamma_{\min }}\right)^{n-1}\left(\pi_{34} C_{a p D}+\pi_{35} C_{a p D}^{2}+\pi_{36} C_{a p D}^{3}\right)\right) .
$$

Here

$$
\begin{aligned}
\frac{\gamma}{\gamma_{\min }}= & \left(\frac{3 \pi_{31}+1}{4 \pi_{31}}\right)^{\frac{\pi_{31}}{\pi_{31}-1}} \frac{1}{\pi_{25}} \frac{k_{w D}}{R_{k D} \mu_{a D}} \\
& \times \sqrt{\frac{\pi_{32}^{2}\left(\frac{\partial p_{a D}}{\partial x_{D}}\right)^{2}+\left(\frac{\pi_{32}}{\pi_{4}}\right)^{2}\left(\frac{\partial p_{a D}}{\partial y_{D}}\right)^{2}+\left(\pi_{32} \pi_{5}\right)^{2}\left(\frac{\partial p_{a D}}{\partial z_{D}}\right)^{2}+\left(\pi_{32} \pi_{5} \pi_{16}\right)^{2} \rho_{a D}^{2}}{\frac{\pi_{33}}{\pi_{25}} \frac{0.5 k_{w D} \phi_{D} \bar{s}_{a}}{R_{k D}}+\frac{\pi_{33} \pi_{10}}{\pi_{25}} \frac{0.5 k_{w D} \phi_{D}}{R_{k D}}}}
\end{aligned}
$$

in which

$$
R_{k D}=\frac{1}{\pi_{25}}+\left(1-\frac{1}{\pi_{25}}\right) \frac{\pi_{28} C_{a p D}}{1+\pi_{28} C_{a p D}} .
$$

Now, we get a complete set of scaling criteria of polymer flooding including 37 dimensionless parameters as follows:

$$
\begin{aligned}
& \pi_{1}=\frac{K_{c w o}}{K_{\text {row }}}, \quad \pi_{2}=\frac{K_{o}}{K_{c w o}}, \quad \pi_{3}=\frac{K_{w}}{K_{\text {row }}}, \quad \pi_{4}=\frac{y_{R}}{x_{R}}, \quad \pi_{5}=\frac{x_{R}}{z_{R}}, \quad \pi_{6}=\frac{x_{p}}{x_{R}}, \\
& \pi_{7}=\frac{y_{p}}{y_{R}}, \quad \pi_{8}=\frac{r_{e o}}{x_{R}}, \quad \pi_{9}=\frac{r_{o}}{x_{R}}, \quad \pi_{10}=\frac{s_{c w}}{\Delta s}, \quad \pi_{11}=\frac{s_{r o}}{\Delta s}, \quad \pi_{12}=\frac{s_{o i}-s_{r o}}{\Delta s}, \\
& \pi_{13}=\frac{\sigma \sqrt{\phi_{0} / K} \cos \theta K_{\text {row }} h}{q_{I} \mu_{w}}, \quad \pi_{14}=\frac{\mu_{o}}{\mu_{w}}, \quad \pi_{15}=\frac{\rho_{o 0}}{\rho_{a 0}}, \quad \pi_{16}=\frac{K_{\text {row }} h}{q_{I} \mu_{w}} \rho_{a 0} g z_{R}, \\
& \pi_{17}=\frac{C_{o} q_{I} \mu_{w}}{K_{\text {row }} h}, \quad \pi_{18}=\frac{C_{a} q_{I} \mu_{w}}{K_{\text {row }} h}, \quad \pi_{19}=\frac{C_{\phi} q_{I} \mu_{w}}{K_{\text {row }} h}, \quad \pi_{20}=\frac{p_{a 0} K_{\text {row }} h}{q_{I} \mu_{w}}, \\
& \pi_{21}=\frac{p_{o 0} K_{\text {row }} h}{q_{I} \mu_{w}}, \quad \pi_{22}=\frac{p_{w f} K_{\text {row }} h}{q_{I} \mu_{w}}, \quad \pi_{23}=\frac{p_{o i} K_{\text {row }} h}{q_{I} \mu_{w}}, \quad \pi_{24}=J\left(\bar{s}_{w}\right), \\
& \pi_{25}=R_{k \max }, \quad \pi_{26}=\frac{\rho_{r} b \hat{C}_{a d \text { max }}}{\phi_{0} \Delta s}, \quad \pi_{27}=\frac{\rho_{r} b \hat{C}_{a d \max }}{\Delta s}, \quad \pi_{28}=b C_{a w p}, \\
& \pi_{29}=\frac{D_{0} \Delta s h}{q_{I} F}, \quad \pi_{30}=\frac{\delta d_{p} \phi_{0} \Delta s}{R_{k \max } x_{R}}, \quad \pi_{31}=n, \quad \pi_{32}=\frac{q_{I}}{h x_{R} \gamma_{\min } \sqrt{k_{\text {row }}},} \\
& \pi_{33}=c^{\prime} \phi_{0} \Delta s, \quad \pi_{34}=A_{p 1} C_{a w p}, \pi_{35}=A_{p 2} C_{a w p}^{2}, \quad \pi_{36}=A_{p 3} C_{a w p}^{3}, \quad \pi_{37}=\frac{C_{a p i}}{C_{a w p}} .
\end{aligned}
$$

From the physical point of view, what these dimensionless parameters mean are as follows:

$\pi_{1}$ - the ratio of the oil permeability under the condition of the irreducible water saturation to the water permeability under the condition of the residual oil saturation.

$\pi_{2}, \pi_{3}$ - the dimensionless permeability of water and oil phase, respectively.

$\pi_{4}, \pi_{5}, \pi_{6}, \pi_{7}, \pi_{8}, \pi_{9}$ - the similarities of geometry, well position and radius, respectively. $\pi_{10}, \pi_{11}$ - the ratios of the irreducible water saturation and the residual oil saturation to the mobile oil saturation, respectively. 
$\pi_{12}$ - the reduced initial oil saturation.

$\pi_{13}$ - can be rewritten as

$$
\frac{\sigma / \sqrt{K / \phi_{0}}}{q_{I} \mu_{w} /\left(K_{\text {row }} h\right)} .
$$

Here $\sqrt{K / \phi_{0}}$ means the average pore radius, and hence $\sigma / \sqrt{K / \phi_{0}}$ denotes the capillary force in the pore of this radius. $q_{I} \mu_{w} /\left(K_{\text {row }} h\right)$ is equivalent to the pressure difference $\Delta p$ induced by the injection rate $q_{I}$ in the reservoir. Consequently, $\pi_{13}$ implies the relative importance between the capillary force and the reservoir pressure difference.

$\pi_{14}, \pi_{15}$ - the viscosity and density ratios of water to oil, respectively.

$\pi_{16}$ - Is equivalent to $\frac{\rho_{w 0} g z_{R}}{\Delta p}$, indicating the relative importance of the gravity and the driving force.

$\pi_{17}, \pi_{18}, \pi_{19}$ - can be uniformly rewritten in the same form as follows

$$
\frac{C_{l} q_{I} \mu_{w}}{K_{\text {row }} h} \propto C_{l} \Delta p,(l=o, a, \phi)
$$

Clearly, they respectively denote the relative volume variation ratios of oil, water, and rock caused by the reservoir pressure.

$\pi_{20}, \pi_{21}, \pi_{22}, \pi_{23}$ - can be uniformly rewritten in the same form of

$$
\frac{p_{i} K_{\text {row }} h}{q_{I} \mu_{w}} \propto \frac{p_{i}}{\Delta p} \quad(i=a 0, o 0, w f, o i) .
$$

Apparently, they denote the respective ratios of the reference pressure of oil and water, the bottom pressure of the production well, and the initial pressure to the reservoir pressure difference.

$\pi_{24}$ - the dimensionless capillary force function.

$\pi_{25}$ - the maximum permeability reduction factor.

$\pi_{26}, \pi_{27}, \pi_{28}$ - the rock characteristics related to adsorption behavior.

$\pi_{29}$ - this can be rewritten as

$$
\frac{D_{0} \Delta s h}{q_{I} F} \propto \frac{D_{0}}{q_{I} / h},
$$

in which $q_{I} / h$ indicates the sweep area of polymer solution injected per unit time. So, it denotes the area ratio between the molecular diffusion and the sweep area.

$\pi_{30}$ - can be rewritten as

$$
\frac{\delta d_{p} \phi_{0} \Delta s}{R_{k \max } x_{R}} \propto \frac{\delta d_{p}}{x_{R}},
$$

indicating the ratio of the mean particle diameter in porous media to the reservoir dimension.

$\pi_{31}$ - the shear rate exponent, expressing the deviation of polymer solution from the Newtonian fluid.

$\pi_{32}$ - is equivalent to 


$$
\frac{q_{I} /\left(h x_{R} \sqrt{k_{\text {row }}}\right)}{\gamma_{\min }},
$$

in which the numerator means the shear rate of the polymer solution in formation. Consequently, it indicates the ratio of the shear rate to the minimum shear rate within the pseudoplastic polymer solution.

$\pi_{33}$ - the tortuosity attributed to rock characteristics.

$\pi_{34}, \pi_{35}, \pi_{36}$ - the dimensionless parameters in the viscosity expression of the polymer solution.

$\pi_{37}$ - the initial concentration of the polymer solution.

At the same spatial and temporal point $\left(x_{D}, y_{D}, z_{D}, t_{D}\right)$ in the model and the prototype, with all above dimensionless parameters kept identical, the dimensionless dependent variables $\bar{s}_{a}, \bar{s}_{o}, p_{a D}, p_{o D}$, and $C_{a p D}$ are equal too.

\section{Numerical Approach of Sensitivity Analysis of the Dimensionless Parameters}

It is not realistic for us to estimate the role of each dimensionless parameter on experimental results by both theory and physical simulation due to too many dimensionless parameters for polymer flooding. Therefore, a numerical approach to quantify this effect is proposed in our previous work for water flooding reservoir (Bai et al. 2005). The quantitative effect of each dimensionless parameter is represented by a sensitivity factor defined as follows.

The sensitivity factor $S_{i}$ of the dimensionless parameter $\pi_{i}$ of a polymer flooding is defined as:

$$
S_{i}=\frac{\partial\left[f\left(\pi_{1}, \pi_{2}, \ldots, \pi_{N}\right) / f_{p}\right]}{\partial\left(\pi_{i} / \pi_{i p}\right)}(i=1,2 \cdots N) .
$$

This means the relative variation ratio of the target function with respect to that of dimensionless parameter $\pi_{i}$. Here, $f\left(\pi_{1}, \pi_{2}, \ldots, \pi_{N}\right)$ denotes a target function concerned in the experiment. It can be pressure, saturation, oil recovery, etc. In polymer flooding, we mainly concern the oil recovery, so the target function can be expressed as

$$
f\left(\pi_{1}, \pi_{2}, \ldots, \pi_{N}\right)=\int_{0}^{T_{D}} \eta\left(\pi_{1}, \pi_{2}, \ldots, \pi_{N}, t_{D}\right) d t_{D},
$$

where $\eta\left(\pi_{1}, \pi_{2}, \ldots, \pi_{N}, t_{D}\right)$ represents the oil recovery curve, $T_{D}$ the dimensionless time span of polymer flooding when the water ratio in the production well is up to $99 \%$. In our numerical approach, the sensitivity factor is given by

$$
S_{i}=\Delta f /\left|w_{i}\right|
$$

in which

$$
\Delta f=\frac{\int_{0}^{T_{D}}\left|\eta_{m}\left(\pi_{1 m}, \pi_{2 m} \ldots, \pi_{i m}, \ldots, \pi_{N m}, t_{D}\right)-\eta_{p}\left(\pi_{1 p}, \pi_{2 p}, \ldots, \pi_{N p}, t_{D}\right)\right| d t_{D}}{\int_{0}^{T_{D}} \eta_{p}\left(\pi_{1 p}, \pi_{2 p}, \ldots, \pi_{N p}, t_{D}\right) d t_{D}},
$$

and

$$
w_{i}=\frac{\pi_{i m}-\pi_{i p}}{\pi_{i p}} .
$$


Table 1 Main physical variables for polymer flooding

\begin{tabular}{|c|c|c|c|}
\hline Physical variable & Value & Physical variable & Value \\
\hline Length $l(\mathrm{~m})$ & 140 & Density of rock $\rho_{r}\left(\mathrm{~kg} / \mathrm{m}^{3}\right)$ & $2.5 \mathrm{E}+3$ \\
\hline Width $w(\mathrm{~m})$ & 140 & $\begin{array}{l}\text { Bottom pressure of the production } \\
\text { well } p_{w f}(\mathrm{~Pa})\end{array}$ & $10 \mathrm{E}+6$ \\
\hline Thickness $h(\mathrm{~m})$ & 10 & Injection rate $q_{I}\left(\mathrm{~m}^{3} / \mathrm{s}\right)$ & $8 \mathrm{E}-3$ \\
\hline $\begin{array}{l}\text { Density of oleic phase under a given con- } \\
\text { dition } \rho_{o 0}\left(\mathrm{~kg} / \mathrm{m}^{3}\right)\end{array}$ & 800 & $\begin{array}{l}\text { Maximum permeability reduction fac- } \\
\text { tor } R_{k} \max \end{array}$ & 1.5 \\
\hline Shear rate exponent $n$ & 0.68 & Well radius $r_{e o}(\mathrm{~m})$ & 0.1 \\
\hline Initial oleic pressure $p_{o i}(\mathrm{~Pa})$ & $12 \mathrm{E}+6$ & $\begin{array}{l}\text { Compressibility of aqueous phase } C_{a} \\
\left(\mathrm{~Pa}^{-1}\right)\end{array}$ & $5 \mathrm{E}-10$ \\
\hline $\begin{array}{l}\text { Density of oleic phase under a given con- } \\
\text { dition } \rho_{w 0}\left(\mathrm{~kg} / \mathrm{m}^{3}\right)\end{array}$ & 1000 & $\begin{array}{l}\text { Maximum adsorption amount per unit } \\
\text { mass of rock } \hat{C}_{a d \max }(\mathrm{kg} / \mathrm{kg})\end{array}$ & $3 \mathrm{E}-6$ \\
\hline Compressibility of oleic phase $C_{o}\left(\mathrm{~Pa}^{-1}\right)$ & $8 \mathrm{E}-10$ & Parameter in viscosity equation $A_{p 1}$ & 1.02 \\
\hline Langmuir adsorption constant $b$ & 0.12 & Parameter in viscosity equation $A_{p 2}$ & 0.18 \\
\hline Compressibility of rock $C_{\phi}\left(\mathrm{Pa}^{-1}\right)$ & $6 \mathrm{E}-10$ & Parameter in viscosity equation $A_{p 3}$ & 0.63 \\
\hline Porosity under a given condition $\phi_{0}$ & 0.25 & $\begin{array}{l}\text { Molecular diffusion coefficient } D_{0} \\
\left(\mathrm{~m}^{2} / \mathrm{s}\right)\end{array}$ & $1.346 \mathrm{E}-9$ \\
\hline Viscosity of water $\mu_{w}$ (Pa.s) & $1 \mathrm{E}-3$ & $\begin{array}{l}\text { The formation electrical resistivity } \\
\text { factor } F\end{array}$ & 5.88 \\
\hline Viscosity of oil $\mu_{o}$ (Pa.s) & $5 \mathrm{E}-3$ & Mean particle diameter $d_{p}(\mathrm{~m})$ & $8.57 \mathrm{E}-4$ \\
\hline
\end{tabular}

the subscripts $m$ and $p$ denote the model and prototype, respectively. Apparently, we can conveniently exhibit the importance degree of each dimensionless parameter by the value of the sensitivity factor.

For a given polymer flooding reservoir, the physical variables are shown in Table 1. Set the deviation coefficient $w_{i}$ of each dimensionless parameter to be $1 \%$, respectively, except for those representing the similarity of well positions, and keep the others identical between the model and the prototype. The governing equations are discretized using a finite difference scheme and solved by the conventional implicit pressure-explicit saturation (Bai et al. 2005; Khalid and Antonin 1979) and implicit concentration method (Cui and Luan 1997). The convection term is discretized using an upwind scheme and the diffusion term with a second-order central difference scheme. The grid scale and time scale are both dimensionless scales in the numerical scheme in order to correspond to the dimensionless governing equations. With an amount of pilot computation, we find that the mesh scales in $x, y$, and $z$ directions equal $0.05,0.05$, and 0.2 , respectively and time scales equal $2.586381 \mathrm{e}-4$ can satisfy our required precision. Then, the sensitivity factors can be obtained and are listed in Table 2.

We can see from Table 2 that the orders of the sensitivity factors generally range from $10^{-5}$ to $10^{0}$ for polymer flooding. Apparently, the larger the sensitivity factor, the more important the corresponding dimensionless parameter. If we choose the dimensionless parameters with a sensitivity factor larger than $10^{-1}$ as the dominant ones, they look like:

$$
\frac{K_{c w o}}{K_{\text {row }}}, \frac{K_{o}}{K_{\text {cwo }}}, \frac{K_{w}}{K_{\text {row }}}, \frac{s_{o i}-s_{\text {ro }}}{\Delta s}, \frac{\mu_{o}}{\mu_{w}}, \frac{\rho_{o 0}}{\rho_{a 0}}, n .
$$

These imply that the ratio of the oil permeability under the condition of the irreducible water saturation to the water permeability under the condition of the residual oil saturation, the 
Table 2 The sensitivity factors

\begin{tabular}{llllllll}
\hline$\pi_{i}$ & 1 & 2 & 3 & 4 & 5 & 9 & 10 \\
$\mathrm{~S}_{i}$ & $2.226 \mathrm{e}-1$ & $2.226 \mathrm{e}-1$ & $2.483 \mathrm{e}-1$ & $2.098 \mathrm{e}-2$ & $6.437 \mathrm{e}-2$ & $1.053 \mathrm{e}-3$ & $6.272 \mathrm{e}-3$ \\
$\pi_{i}$ & 11 & 12 & 13 & 14 & 15 & 16 & 17 \\
$\mathrm{~S}_{i}$ & $2.738 \mathrm{e}-3$ & $1.342 \mathrm{e}-0$ & $7.723 \mathrm{e}-4$ & $2.227 \mathrm{e}-1$ & $2.622 \mathrm{e}-1$ & $3.355 \mathrm{e}-2$ & $2.352 \mathrm{e}-3$ \\
$\pi_{i}$ & 18 & 19 & 20 & 21 & 22 & 23 & 24 \\
$\mathrm{~S}_{i}$ & $1.734 \mathrm{e}-3$ & $1.650 \mathrm{e}-3$ & $6.436 \mathrm{e}-4$ & $1.450 \mathrm{e}-2$ & $2.489 \mathrm{e}-2$ & $1.587 \mathrm{e}-2$ & $7.723 \mathrm{e}-4$ \\
$\pi_{i}$ & 25 & 26 & 27 & 28 & 29 & 30 & 31 \\
$\mathrm{~S}_{i}$ & $2.125 \mathrm{e}-2$ & $1.053 \mathrm{e}-4$ & $5.851 \mathrm{e}-5$ & $6.506 \mathrm{e}-3$ & $4.681 \mathrm{e}-5$ & $4.681 \mathrm{e}-5$ & $9.163 \mathrm{e}-2$ \\
$\pi_{i}$ & 32 & 33 & 34 & 35 & 36 & 37 & $1.428 \mathrm{e}-2$ \\
$\mathrm{~S}_{i}$ & $2.223 \mathrm{e}-2$ & $1.115 \mathrm{e}-2$ & $4.695 \mathrm{e}-2$ & $5.933 \mathrm{e}-3$ & $1.708 \mathrm{e}-2$ & & \\
\hline
\end{tabular}

dimensionless permeability of water and oil, the density and viscosity ratios of water and oil, the reduced initial oil saturation, and the shear rate exponent of polymer solution are the most important parameters and should be scaled precisely in the polymer flooding modeling. These results just reflect the nature of the polymer flooding modeling. Among the attributes related to a porous medium, the permeability exerts more influence on flows than others do. Among the properties of fluids, the density and viscosity are more important. And the shear rate exponent is a dominant one because it represents the non-Newtonian behavior of the polymer solution and just reflects the particular driving mechanism of polymer flooding. In addition, the dimensionless parameter $\pi_{5}=x_{R} / z_{R}$ scaling the vertical dimension exerts considerable influence on experiment results although its sensitivity factor is slightly less than that of those dominant ones. This is because the polymer flooding enhances oil recovery by enlarging the volumetric sweep efficiency, which is influenced by geometry. Other factors, such as the gravity and the capillary forces, the compressibility, adsorption, diffusion, and dispersion, may be relaxed if they contradict the dominant ones. The error induced by relaxing the secondary dimensionless parameters are far less than the dominant ones.

\section{The Effect of Physical Variables on the Sensitivity}

The above results are applied to the polymer flooding with the physical variables listed in Table 1. In fact, the dominant dimensionless parameters may vary with the physical variable range shown in our previous work (Bai et al. 2006). However, it is impossible to analyze the effect of each variable range on the dominance degree of all the dimensionless parameters due to the reservoirs are diverse. On the other hand, the amount of variable values within the variable range is theoretically infinite and therefore it is also impossible to analyze each value effect within the variable range. We just select some typical values within the practical field variable range, which can represent the typical conditions. In this section, we focus on the main physical variables effect. The variation tendency of the dominance degree of the dimensionless parameters with viscosity, injection rate and permeability is investigated.

\subsection{Viscosity Effect}

Selecting 2.5mPa.s, $5 \mathrm{mPa} . \mathrm{s}$, and 50mPa.s as examples of low, moderate, and high oil viscosity reservoirs and keeping other physical variables unchanged, we obtain the variation tendency 


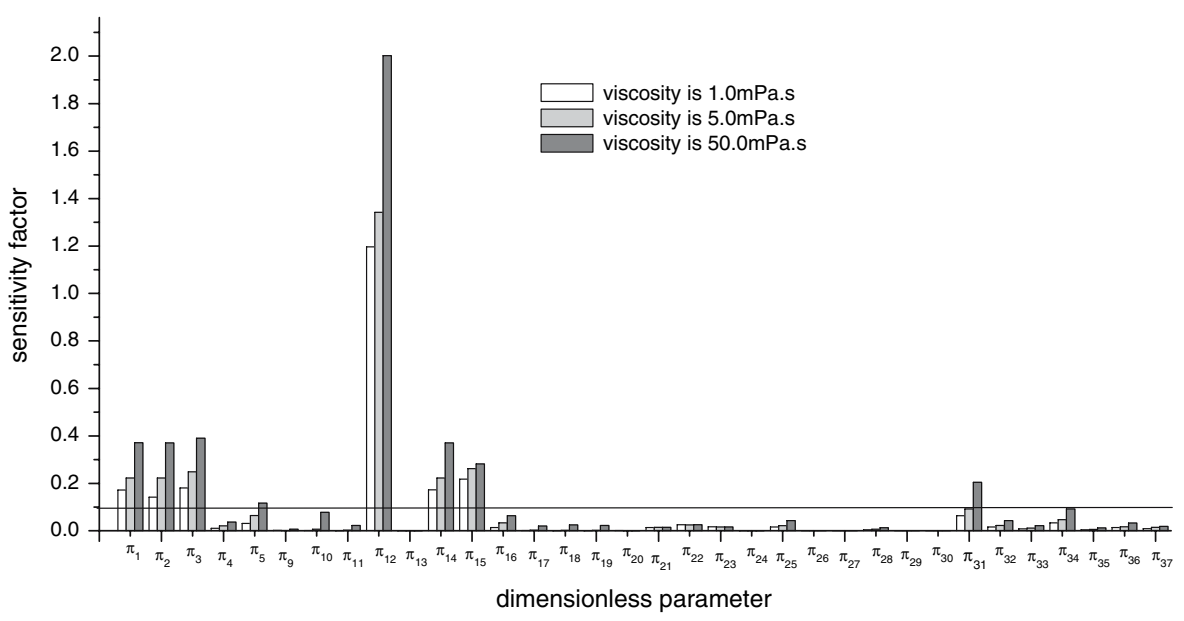

Fig. 1 The effect of oil viscosity on the dominance degree of the dimensionless parameters

of the dominance degree with oil viscosity shown in Fig. 1. A line of 0.1 demarcating the sensitivity factor level of the dominant and secondary dimensionless parameters is also drawn in Fig. 1. That is to say, the dimensionless parameter is regarded as a dominant one if its sensitivity factor is greater than 0.1 . Otherwise it is deemed as a secondary one. This rule also applies in the following texts. We can see from Fig. 1 that the dominant dimensionless parameters are $\pi_{1}, \pi_{2}, \pi_{3}, \pi_{12}, \pi_{14}$, and $\pi_{15}$ for a low oil viscosity reservoir, $\pi_{1}, \pi_{2}, \pi_{3}, \pi_{12}, \pi_{14}, \pi_{15}$, and $\pi_{31}$ for a moderate oil viscosity reservoir, and $\pi_{1}, \pi_{2}, \pi_{3}, \pi_{5}, \pi_{12}, \pi_{14}, \pi_{15}, \pi_{31}$, and $\pi_{34}$ for a high oil viscosity reservoir. In addition, $\pi_{10}$ almost approaches the dominant one in the oil high viscosity reservoir.

Obviously, $\pi_{5}$ plays a greater important role with oil viscosity and becomes a dominant one in the high viscosity reservoir. Polymer flooding can largely improve oil recovery efficiency than water flooding. With low oil viscosity, we presumably consider that the oil recovery efficiency is very high. And the formation not swept by the polymer solution is less than that in the case of high oil viscosity. Therefore, whether the geometry is satisfied or not, has less effect on oil recovery efficiency. On the contrary, high oil viscosity will lead to relatively more formation not swept. In this case, the geometry will affect the oil recovery efficient.

$\pi_{10}$ exerts more and more influence on the flows with oil viscosity. This is simply because increasing the oil viscosity reduces the oil recovery, which means the oil captured in the reservoir increases. Thus, the mobile oil volume reduces, indicating the increasing of the residual oil and irreducible water saturation. Therefore, $\pi_{10}$ has an evident effect on the mobile oil volume and dramatically affects the oil recovery. On the contrary, in a low oil viscosity reservoir, the high oil recovery means less oil staying in the reservoir and a more mobile oil volume. The less the residual oil and irreducible water saturation are, the less is the effect of them on the mobile oil volume and the oil recovery.

With the increasing of oil viscosity, $\pi_{31}$ plays a more and more important role and becomes dominant in moderate and high oil viscosity reservoirs. The reason is that $\pi_{31}$ is the shear rate exponent, which represents the non-Newtonian behavior of polymer solution. It affects the viscosity of the polymer solution. Generally, the viscosity ratio of the oleic to aqueous phases always has a large effect on oil recovery. The effect of $\pi_{31}$ on oil recovery is reflected by its effect on the viscosity ratio of the oleic to aqueous phases. With the increasing of oil 


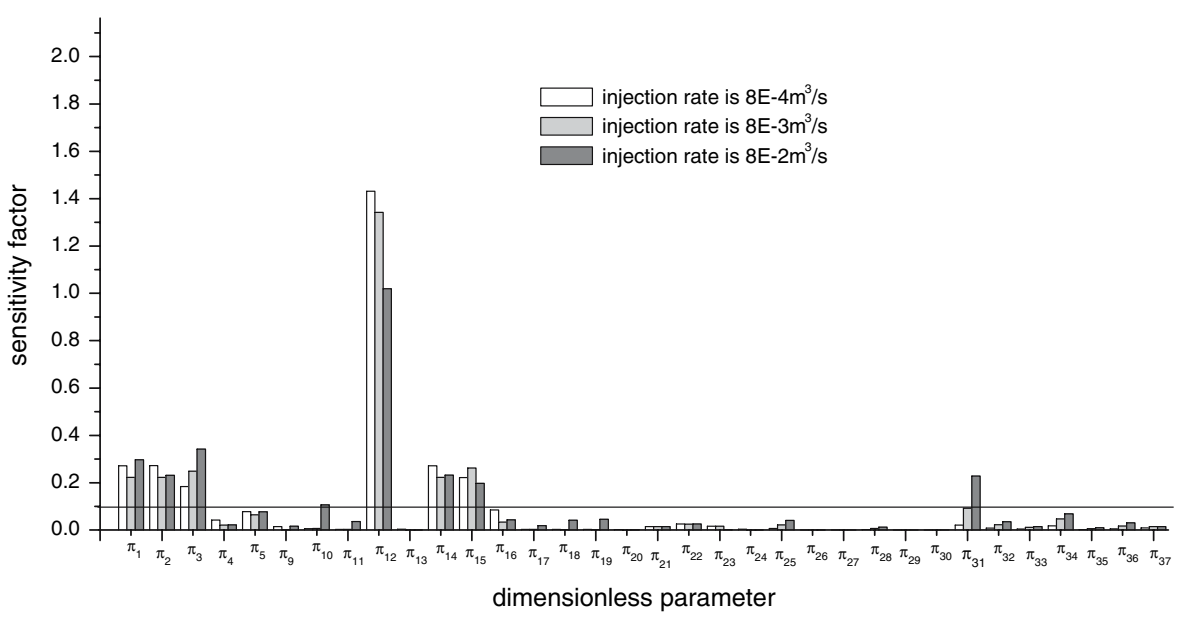

Fig. 2 The effect of the injection rate on the dominance degree of the dimensionless parameters

viscosity, the viscosity ratio of the oleic to aqueous phases increases too. Therefore, the effect of the viscosity of the polymer solution on the viscosity ratio in the moderate and high oil viscosity cases is larger than that in low oil viscosity. Hence, $\pi_{31}$ becomes dominant in the moderate and high viscosity reservoirs.

Finally, we find out that $\pi_{34}$ turns to be dominant in high oil viscosity. Since $\pi_{34}$ denotes the coefficient of one-order term in the dimensionless viscosity equation of the polymer solution, its variation can largely affect the viscosity. In a high viscosity reservoir, the variation of $\pi_{34}$ exerts more influence on the viscosity ratio of the oleic to aqueous phases and thus affects oil recovery than that in the low viscosity case, as explained to $\pi_{31}$.

\subsection{Injection Rate Effect}

During the different stages of reservoir development, the injection rate is adjusted to fit the formation characteristics. This variation changes the distribution of pressure in the formation, and then a new balance of forces will be established to adapt the injection rate. We select $8 \mathrm{E}-4 \mathrm{~m}^{3} / \mathrm{s}, 8 \mathrm{E}-3 \mathrm{~m}^{3} / \mathrm{s}, 8 \mathrm{E}-2 \mathrm{~m}^{3} / \mathrm{s}$ as low, moderate, and high injection rates. The variation tendency of the dominance degree with the injection rate is shown in Fig. 2. We can see that $\pi_{1}, \pi_{2}, \pi_{3}, \pi_{12}, \pi_{14}, \pi_{15}$, and $\pi_{16}$ are the dominant dimensionless parameters for a low injection rate, $\pi_{1}, \pi_{2}, \pi_{3}, \pi_{12}, \pi_{14}, \pi_{15}$, and $\pi_{31}$ for a moderate injection rate, and $\pi_{1}, \pi_{2}, \pi_{3}, \pi_{10}, \pi_{12}, \pi_{14}, \pi_{15}$, and $\pi_{31}$ for a high injection rate.

$\pi_{10}$ exerts greater influence on flows with the injection rate, and it turns to a dominant dimensionless parameter at a high injection rate. The reason is that on the one hand, a high injection rate increases the shear rate, causing the reduction of the viscosity of polymer solution. On the other hand, the high injection rate causes early water breakthrough and flooding. Both lower the oil recovery within a certain development time span. For the low oil recovery reservoir, the variation of the irreducible water saturation can lead to a relatively larger variation of oil recovery. The reason is similar, as the explanation in Sect. 5.1.

$\pi_{16}$ plays a more and more important role with the reduction of the injection rate and becomes dominant in a low injection rate. The reason is that it denotes the relative importance between the gravity force and the driving force. With the reduction of the injection 


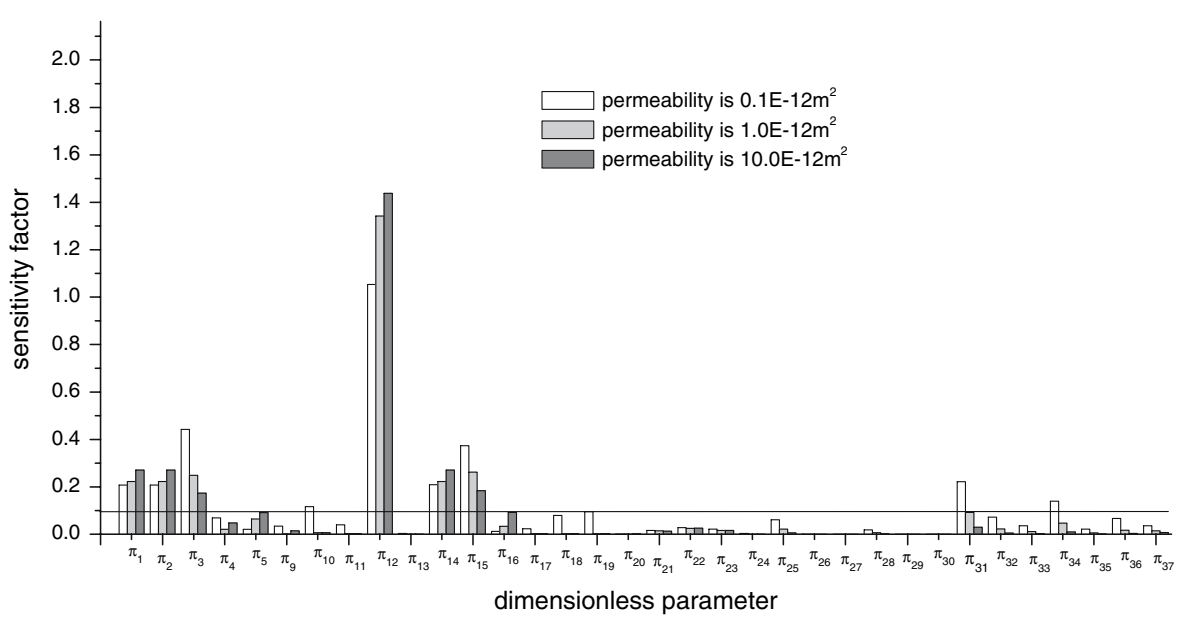

Fig. 3 The effect of the permeability on the dominance degree of the dimensionless parameters

rate, the driving force decreases too. Therefore, the gravity force exerts a more and more important influence on vertical flows and cannot be neglected.

$\pi_{31}$ may become dominant at a high injection rate. The reason is that the viscosity of polymer solution is related with the shear rate. With the increasing of the injection rate the viscosity decreases and the non-Newtonian behavior is more apparent, which affects the viscosity ratio of the oleic to aqueous phases and thus affects considerably the oil recovery as discussed above.

\subsection{Permeability Effect}

We select $0.1 \mathrm{~d}, 1 \mathrm{~d}$, and $10 \mathrm{~d}$ as the examples of low, moderate, and high permeability reservoirs to study the effect of the permeability on the dominance degree with the other physical variables unchanged. The result is shown in Fig. 3. We can see that the dominant dimensionless parameters are $\pi_{1}, \pi_{2}, \pi_{3}, \pi_{10}, \pi_{12}, \pi_{14}, \pi_{15}, \pi_{19}, \pi_{31}$, and $\pi_{34}$ in a low permeability reservoir, $\pi_{1}, \pi_{2}, \pi_{3}, \pi_{12}, \pi_{14}, \pi_{15}$, and $\pi_{31}$ in the moderate permeability case, whereas $\pi_{1}, \pi_{2}, \pi_{3}, \pi_{5}, \pi_{12}, \pi_{14}, \pi_{15}$, and $\pi_{16}$ for the case of high permeability.

$\pi_{5}$ may turn to a dominant one in high permeability. The reason is that the sweep coefficient enlarges with permeability. In the case of high permeability, whether the geometric similarity is satisfied or not, exerts evident influence on the sweep coefficient, thus affecting the oil recovery.

With the reduction of the permeability, $\pi_{10}$ turns to be dominant. This can be explained as follows. With the reduction of the permeability, the resistance force of flow increases and the oil recovery reduces. Therefore, the variation of $\pi_{10}$, namely, the variation of the mobile oil volume evidently exerts influences on the flow channel, which is small in the low permeability case. In a low oil recovery reservoir, $\pi_{10}$ has a great effect, as mentioned above.

$\pi_{19}$ exerts more and more influence on the reservoir flows with the reduction of the permeability. The reason is that the pressure in the formation increases with the reduction of the permeability, which leads to a relatively larger volume variation of rock. Therefore, the compressibility should be considered in low permeability.

$\pi_{31}$ turns from a secondary one in high permeability reservoir to a dominant one in the low permeability case. The reason is that the shear rate in low permeability is larger than that 
in the high permeability case i.e., the non-Newtonian behavior in low permeability is more apparent. Therefore, with the same variation of $\pi_{31}$, the viscosity variation of the polymer solution in the low permeability is larger than that in the high permeability. This leads to considerable variation of the viscosity ratio of oil to phase in the low permeability case, thus evidently affecting oil recovery as mentioned above.

With the reduction of the permeability, $\pi_{34}$ exerts a greater influence and becomes dominant. The reason is that in the case of low permeability, the shear rate increases and thus considerably affects the viscosity. Therefore, $\pi_{34}$ exerts more influence on the viscosity ratio of the oleic to aqueous phases in low permeability than that in the high permeability case, thus more apparently affecting the oil recovery.

\section{Conclusions}

The authors of this article have derived a set of scaling criteria for a polymer flooding reservoir, taking into consideration gravity and capillary forces, compressibility of water, oil, and rock, non-Newtonian behavior of polymer solution, absorption, dispersion, and diffusion. A numerical approach to quantify the dominance degree of each dimensionless parameter is proposed. By this approach, the sensitivity factor of each dimensionless parameter is evaluated. The results show that in polymer flooding, the orders of the sensitivity factor generally range from $10^{-5}$ to $10^{0}$.

The dominant dimensionless parameters for a polymer flooding are generally the ratio of the oil permeability under the condition of the irreducible water saturation to the water permeability under the condition of residual oil saturation, density, and viscosity ratios between water and oil, reduced initial water saturation, and the shear rate exponent. Other factors, such as the gravitational and the capillary forces, compressibility, adsorption, diffusion, and dispersion, may be relaxed if they contradict the dominant factors.

The effects of some physical variables, such as oil viscosity, injection rate, and permeability, on the dominance degree of the dimensionless parameters, are analyzed. The results show that the dominant dimensionless parameters differ from case to case with different physical variables and operation conditions. Please notice that the dominant dimensionless parameters and their variation tendency with some physical variables are based on the definition with the oil recovery as the target function. As the target function changes, the results may be different. In this article, we have investigated the effect of three main physical variables i.e., viscosity, injection rate, and permeability. The other effects on the dominance degree of dimensionless parameters are our next task. The limitation in the investigation of the effect of physical variables on the sensitivity is that we just select some typical values within the practical field variable range. Theoretically, the amount of variable values within the variable range is infinite.

Acknowledgements The writers greatly appreciate the financial support of the National Basic Research Program of China (Grant No. 2005CB221300), and the Innovative Project of the Chinese Academy of Sciences (Grant No. KJCX-SW-L08).

\section{References}

Bai, Y., Li, J., Zhou, J.: Sensitivity analysis of dimensionless parameters for physical simulation of waterflooding reservoir. Sci. China Ser. E. 48(4), 441-453 (2005) 
Bai, Y., Li, J., Zhou, J.: Effect of physical parameter range on dimensionless variable sensitivity in water flooding reservoirs. Acta Mech. Sinica 22(5), 385-391 (2006)

Delamaide, E., Corlay, P., Wang, D.-M.: Daqing oil field: the success of two pilots initiates first extension of polymer injection in a giant oil field. SPE 27819, Proc. SPErDOE 9th Symp. on EOR. pp. 401-410. Tulsa, 17-20 April (1994)

Cui, C., Luan, Z.: Thermal-polymer flooding study. Acta Petrol. Sin. 18(2), 56-62 (in Chinese) (1997)

Han, D., Yang, C., Zhang, Z.: Recent development of enhanced oil recovery in China. J. Petrol. Sci. Eng. 22, 181-188 (1999)

Islam, M.R., Farouq Ali, S.M.: New scaling criteria for polymer emulsion and foam flooding experiments. J. Can. Petrol. Technol. 28(4), 79-87 (1989)

Islam, M.R., Farouq Ali, S.M.: New scaling criteria for chemical flooding experiments. J. Can. Petrol. Technol. 29(1), 29-36 (1990)

Kevin, C.T., Hisham, A., Nasr, E.-D.: Water-soluble hydrophobically associating polymers for improved oil recovery: a literature review. J. Petrol. Sci. Eng. 19, 265-280 (1998)

Khalid, A., Antonin, S.: Petroleum Reservoir Simulation. Applied Science Publishers, London (1979)

Littmann, W.: Polymer flooding. Oil Industry Press, Beijing (in Chinese) (1991)

Lu, G., Gao, Z., Zhao, X.: Distribution regularities of remaining oil in heterogeneous reservoirs after polymer flooding. Acta Petrol. Sin. 17(4), 55-61 (in Chinese) (1996)

Luis, E.Z., Nestor, V.Q.T., Salvador, P., Jean-Louis, S.: An optimization methodology of alkaline-surfactantpolymer flooding processes using field scale numerical simulation and multiple surrogates. J. Petrol. Sci. Eng. 47, 197-208 (2005)

Perkins, K., Johnston, O.C.: A review of diffusion and dispersion in porous media. Soc. Pet. Eng. J. 3, 7085 (1962)

Pozzi, A.L., Blackwell, R.J.: Design of laboratory models for study of miscible displacement. Soc. Pet. Eng. J. 4, 28-40 (1963)

Prabir, D., Pasa, G.: An optimal viscosity profile in enhanced oil recovery by polymer flooding. Int. J. Eng. Sci. 42, 2029-2039 (2004)

Sedov, L.I.: Similarity and Dimensional Methods in Mechanics. Academic Press, New York (1959)

Shen, P.: Fundamental research on largely enhancing oil recovery. China Basic Sci. 2:9-14 (in Chinese) (2003)

Shen P.: The Theory and Experiment of Water and Oil Flow in Porous Media. Oil Industry Press, Beijing (in Chinese) (2000)

Sorbie, K.S., Parker, A., Clifford, P.J.: Experimental and theoretical study of polymer flow in porous media. SPE 14231, 1-17 (1985)

Wang, D., Cheng, J., Yang, Q.: Viscoelastic polymer solution can enhance the microscopic driving efficiency. Acta Petrol. Sin. 21(5), 45-51 (in Chinese) (2000)

Wang, D.-M., Yue-xing, H., Delamaide, E., Ye, Z.-G., Ha, S., Jiang, X.-C.: Results of two polymer flooding pilots in the central area of Daqing oil field. SPE 26401, Proc. 68th Ann. Tech. Conf. and Exhibition. Houston 3(6), 291-308 (1993)

Wang, X.: Determination of the main parameters in the numerical simulation of polymer flooding. Petrol. Explor. Develop. 3, 69-075 (in Chinese) (1990)

Wang, X., Han, D., Guo, S.: The mechanism of polymer flooding and its mathematical and parametric models. Chin. Sci. Bull. 18, 1713-1715 (in Chinese) (1992)

Wang, X., Han, D.: Mechanism and application of polymer flooding. Acta Petrol. Sin. 15(1), 83-91 (in Chinese) (1994) 